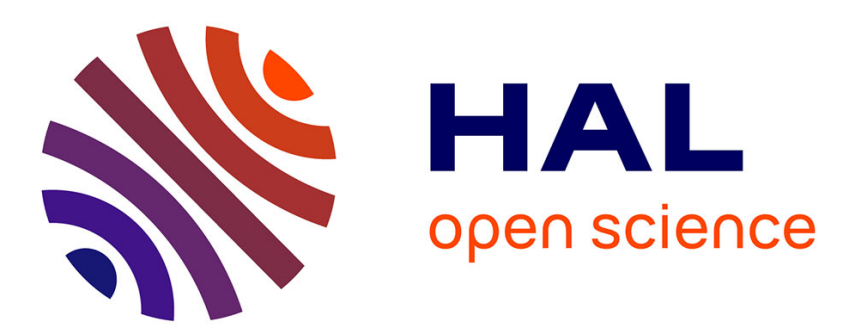

\title{
Graphene on TaC: Air tight protection of a superconducting surface
}

L. Huder, Guy Trambly de Laissardière, G. Lapertot, A.G.M. G M Jansen, C. Chapelier, V.T. Renard

\section{- To cite this version:}

L. Huder, Guy Trambly de Laissardière, G. Lapertot, A.G.M. G M Jansen, C. Chapelier, et al.. Graphene on TaC: Air tight protection of a superconducting surface. Carbon, 2018, 140, pp.592 - 595. 10.1016/j.carbon.2018.09.003 . hal-01877574

\section{HAL Id: hal-01877574 https://hal.science/hal-01877574}

Submitted on 24 Sep 2018

HAL is a multi-disciplinary open access archive for the deposit and dissemination of scientific research documents, whether they are published or not. The documents may come from teaching and research institutions in France or abroad, or from public or private research centers.
L'archive ouverte pluridisciplinaire HAL, est destinée au dépôt et à la diffusion de documents scientifiques de niveau recherche, publiés ou non, émanant des établissements d'enseignement et de recherche français ou étrangers, des laboratoires publics ou privés. 


\title{
Graphene on TaC: air tight protection of a superconducting surface
}

\author{
L. Huder,${ }^{1}$ G. Trambly de Laissardière,${ }^{2}$ G. Lapertot,${ }^{1}$ A. G. M Jansen, ${ }^{1}$ C. Chapelier,${ }^{1}$ and V. T. $\operatorname{Renard}{ }^{1}$ \\ ${ }^{1}$ Univ. Grenoble Alpes, CEA, INAC-PHELIQS, F-38000 Grenoble, France \\ ${ }^{2}$ LPTM, Université de Cergy-Pontoise-CNRS, F-95302 Cergy-Pontoise, France
}

\begin{abstract}
We report on the fabrication of a clean, stable in room atmosphere and superconducting surface consisting of graphene on tantalum carbide $(\mathrm{TaC})$ obtained by high-temperature annealing of a tantalum thin film on SiC. Low temperature scanning tunnelling spectroscopy reveals that the surface is superconducting with a superconducting order parameter up to $1.6 \mathrm{meV}$. In addition, atomically-resolved imaging establishes the chemical inertness of the surface. This result is achieved because few graphene layers protect the surface superconductivity of the buried superconducting TaC formed during the high-temperature annealing.
\end{abstract}

\section{INTRODUCTION}

Since the invention of the scanning tunnelling microscope (STM), graphite has been the substrate of choice for atomically resolved imaging because it offers a surface which is easy-to-produce, atomicallyclean and stable in room atmosphere $e^{1}$. This surface quality has allowed the study of various isolated or self-organized molecular systems ${ }^{2}$. Recently, there has been a surge of interest for the study of magnetic atoms or molecules at the surface of superconductors because these systems are prime candidates to bear topological phases and, in particular, Majorana zero modes (see Ref. ${ }^{[3}$ and references therein for a review). These investigations are challenging because superconductors, like most conductors, oxidize in room atmosphere ${ }^{4}$ which puts stringent constraints on experimental conditions and surface preparation. Surface reactivity is also a concern as reactions between the magnetic adsorbates and the surface could alter the output of the experiments. It would be therefore highly desirable to have a superconducting analogue of HOPG to conduct this research.

The rise of two-dimensional (2D) superconductors has not allowed improvements since no inert 2D superconductor has been isolated yet ${ }^{516}$. Capping 2D superconductors with a protective 2D layer (either graphene or h-BN) has however revealed a fruitful strategy to preserve the structural and electronic properties of those fragile materials 78 . Importantly, superconductivity can still be probed through the capping layer owing to its extreme thinness. Unfortunately, the transfer technique implemented in those studies does not allow the preparation of large surface areas which are more convenient for STM studies. In view of producing large areas, an interesting proposal has been made in Ref. ${ }^{[9}$ where graphene was deposited by CVD on rhenium to protect this conventional superconductor. However, in this study, graphene is strongly hybridized with the substrate and loses both its two-dimensional character and its Dirac electronic properties.

We present here a similar strategy, albeit using graphitization of a superconducting carbide, to get a clean and superconducting surface spanning large area with a relatively large energy gap which would be suitable for easy STM experiments. This result follows from our recent discovery that high temperature annealing of a carbide-forming metal ( $\mathrm{Nb}$ or $\mathrm{Ta}$ ) thin film on silicon carbide $(\mathrm{SiC})$ leads to the formation in a single process step of a superconducting carbide ( $\mathrm{NbC}$ or $\mathrm{TaC}$ ) capped with graphene layers ${ }^{10}$. We have shown by transport measurements that the known strong interaction between graphene and superconducting carbides ${ }^{11}$ allows for the realization of high-quality epitaxial electrical contacts between graphene and the superconducting carbide ${ }^{10}$. In this article, we present a study of surface superconductivity probed by low temperature STM measurements (topography and spectroscopy). It is demonstrated that the surface of the graphene layers on top of $\mathrm{TaC}$ is clean and retains simultaneously a superconducting gap and a Dirac cone which mimics that of graphene despite exposure to ambiant air without further surface preparation.

\section{EXPERIMENTAL AND COMPUTATIONAL PROCEDURES}

\section{A. Sample preparation}

The TaC and graphene films were prepared by depositing a $40 \mathrm{~nm}$ thick layer of tantalum by e-beam evaporation on top of the carbon terminated surface of $4 \mathrm{H}-\mathrm{SiC}$ substrates. The samples were then annealed in a RF-induction furnace inside a graphite crucible under a pressure lower than $10^{-6} \mathrm{mbar}$, following a recipe similar to that for graphene growth on $\mathrm{SiC}^{12}$. The first annealing step ramps the tem- 
a)

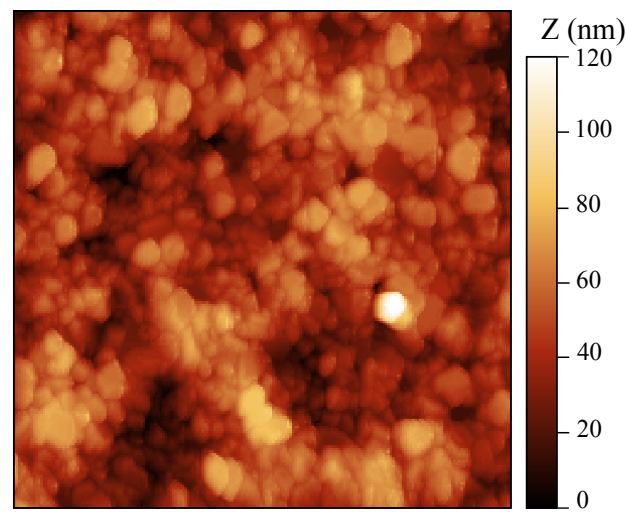

b)

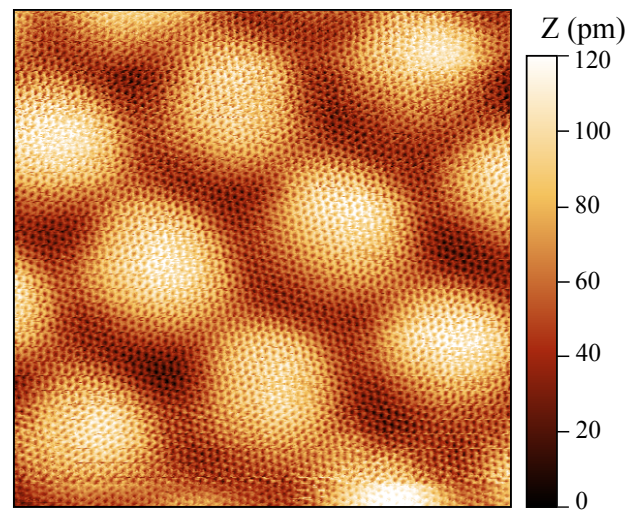

Figure 1. a) A $2 \mu \mathrm{m} \times 2 \mu \mathrm{m}$ AFM image of the surface of a graphitized $\mathrm{TaC}$ sample showing the typical grains. b) A $13 \times 13 \mathrm{~nm}^{2}$ STM topography image of graphene layers on a $\mathrm{TaC}$ grain showing the moiré pattern typical of twisted graphene layers $\left(V_{b}=-4 \mathrm{mV}, I_{t}=1 \mathrm{nA}\right)$ at $4.2 \mathrm{~K}$.

perature to $1140^{\circ} \mathrm{C}$ in 60 minutes and holds this temperature during 30 minutes for degassing. The next temperature ramp is done in 180 minutes up to $1360^{\circ} \mathrm{C}$ and the temperature is then kept stable for 18 minutes. The cooling down to room temperature follows a reversed procedure.

\section{B. STM measurements}

After thermal annealing, the sample remained in air atmosphere for one day before it was loaded without further treatment into a home-made low temperature STM operating down to $1.5 \mathrm{~K}$. Images of the surface topography were acquired in the constant current mode and unless specified recorded at $4.2 \mathrm{~K}$. The spectroscopic measurements of the Local Density of States (LDOS) were performed by measuring the differential conductance $(d I / d V)$ using phase sensitive detection while ramping the tip-sample bias $V_{b}$ at fixed tip height.

\section{Tight binding calculations}

Local density of states (LDOS) are computed by recursion method in real space from a tight-binding (TB) Hamiltonian that has been shown to simulate LDOS in twisted graphene bilayer with strain ${ }^{13}$ and without strain $14 \mid 15$. Only $p_{z}$ orbitals of $\mathrm{C}$ atoms are taken into account since we are interested in what happens around the Fermi level.

The Hamiltonian has the form:

$$
H=\sum_{i} \epsilon_{i}|i\rangle\left\langle i\left|+\sum_{<i, j\rangle} t_{i j}\right| i\right\rangle\langle j|,
$$

where $|i\rangle$ is the $p_{z}$ orbital located at $\vec{r}_{i}$, and $\langle i, j\rangle$ is the sum on index $i$ and $j$ with $i \neq j$. The coupling matrix element, $t_{i j}$, between two $p_{z}$ orbitals of atoms $i$ and $j$, is computed by Slater-Koster formula from the hopping parameters 16 ,

$$
\begin{aligned}
& V_{p p \pi}\left(r_{i j}\right)=-\gamma_{0} \mathrm{e}^{q_{\pi}\left(1-\frac{r_{i j}}{a_{0}}\right)}, \\
& V_{p p \sigma}\left(r_{i j}\right)=\gamma_{1} \mathrm{e}^{q_{\sigma}\left(1-\frac{r_{i j}}{a_{1}}\right)},
\end{aligned}
$$

where $r_{i j}$ is the distance between the two orbitals, $a_{0}=1.418 \AA$ is the nearest neighbor distance within a layer, and $a_{1}=3.349 \AA$ is the interlayer distance. First neighbors interaction in a plane $\gamma_{0}=3.7 \mathrm{eV}$ is taken to recover the Fermi velocity which in graphene equals to $1.09 \times 10^{6} \mathrm{~m} . \mathrm{s}^{-114}$. Second neighbors interaction $\gamma_{0}^{\prime}$ in a plane is set to $0.1 \gamma_{0} \sqrt{17}$ which fixes the value of the ratio $q_{\pi} / a_{0}$ in equation (2). The inter-layer coupling between two $p_{z}$ orbitals in $\pi$ configuration is $\gamma_{1} . \gamma_{1}$ is fixed to obtain a good fit with DFT calculations around the Dirac energy in AA stacking and AB Bernal stacking which results in $\gamma_{1}=0.48 \mathrm{eV}^{16}$. To get $q_{\sigma}$, we choose the same coefficient of the exponential decay for $V_{p p \pi}$ and $V_{p p \sigma}$. All $p_{z}$ orbitals have the same onsite energy $\epsilon_{i}$ equal to the Dirac energy in monolayer graphene. 
a)

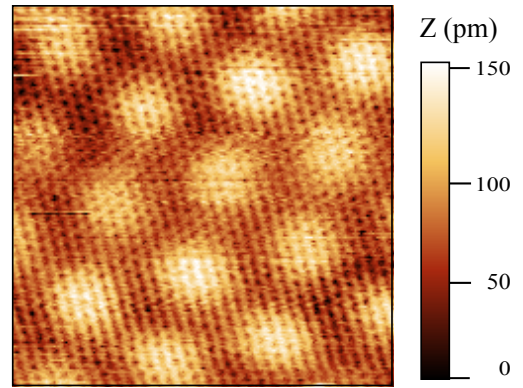

d)

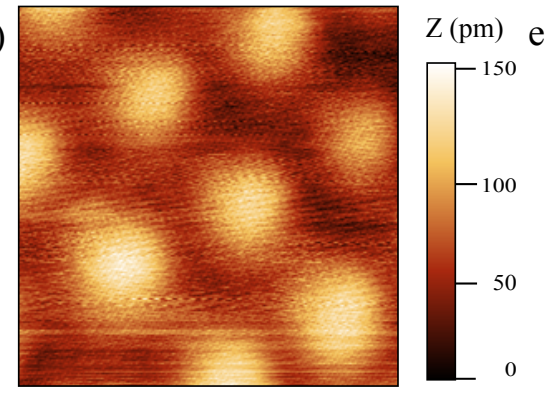

b)
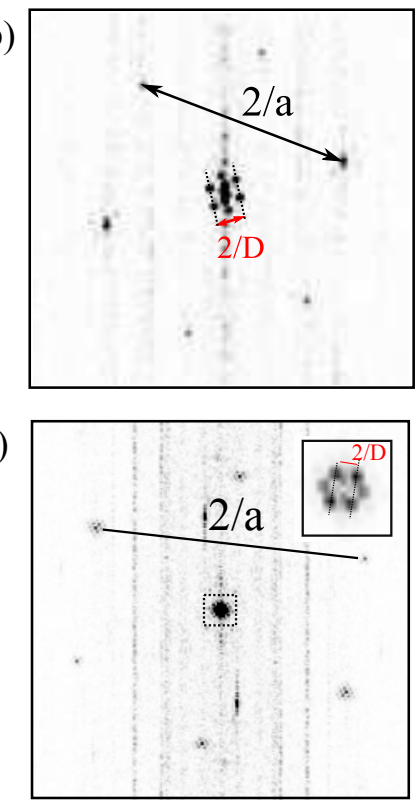

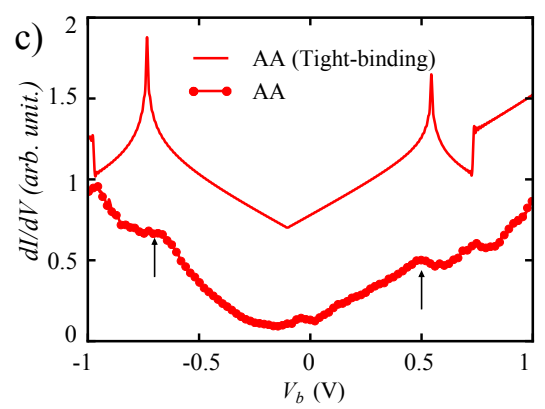

f)

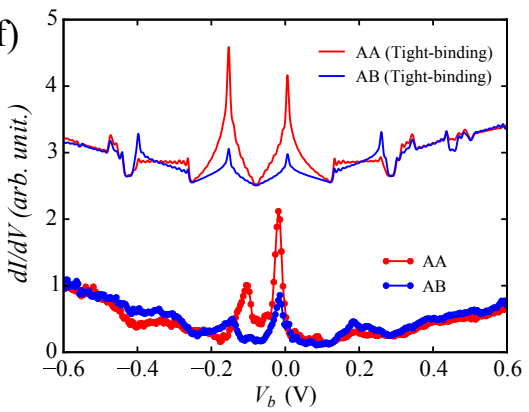

Figure 2. a) A $6 \times 6 \mathrm{~nm}^{2} \mathrm{STM}$ topography image of twisted graphene layers found on a TaC grain $\left(V_{b}=-1 \mathrm{~V}\right.$, $\left.I_{t}=500 \mathrm{pA}\right)$. The rotation angle is $7.25^{\circ}$. b) Fourier transform of the image in panel $(a)$. Arrows indicate the distances from which the graphene lattice parameter $a$ and the moiré period $D$ can be determined. c) LDOS of the grain shown in panel (a). The LDOS is measured by scanning tunnelling spectroscopy (dotted line) with a $6 \mathrm{mV}$ $\mathrm{AC}$ bias modulation and shows van Hove singularities which are independent of the position where the spectrum is measured. The theoretical prediction is shown as a solid line. It has been shifted vertically by 0.75 arbitrary unit. d) A $16 \times 16 \mathrm{~nm}^{2} \mathrm{STM}$ image of rotated graphene layers found on a TaC grain $\left(V_{b}=-1 \mathrm{~V}, I_{t}=1 \mathrm{nA}\right)$. The rotation angle is $2^{\circ}$. e) Fourier transform of the image in panel $(d)$. The inset shows a zoom on the moiré harmonics at the center of the Fourier transform. f) Experimental LDOS acquired in AA (red dotted line) and AB (blue dotted line) regions of the grain presented in panel (d). The measurement was performed with a $6 \mathrm{mV} \mathrm{AC}$ bias modulation. The corresponding theoretical prediction is shown as a solid line. They have been shifted vertically by 2.5 arbitrary unit.

\section{RESULTS AND DISCUSSION}

\section{A. Graphene on TaC: topography and spectroscopy.}

Figure 11 shows a large scale Atomic Force Microscope (AFM) image of the surface of the sample after the thermal annealing. One recognizes the typical granular morphology of $\mathrm{TaC}$ on $\mathrm{SiC}$ with typical grain size $\sim 200 \mathrm{~nm} \frac{10}{}$. The atomically resolved low temperature STM image of Fig 1 $1 \mathrm{~b}$ confirms our previous study in that the grains are covered with graphene $e^{10}$. A clear moiré signal is superposed to the atomic signal indicating that several graphene layers (at least two) are present at the surface. Bright regions in Figs. 1p correspond to regions where the atomic lattices of the two layers are in registry (AA regions) while the lattices are in anti-phase in dark regions (AB regions) 18119 . Figures 1 and 2 show that there is no preferential stacking angle as several moiré lattice parameters $D$ are observed, indicating different rotation angles between the topmost layers. The lattice constant $a$ and moiré periodicity $D$ are found from the Fourier transform (Figs. 2p and d) which allows the determination of the average rotation angle $\theta$ according to $\theta=2 \sin ^{-1}\left(\frac{a}{2 D}\right)$. For instance, the rotation angle is $2.46^{\circ}$ in Fig. 11 $7.25^{\circ}$ in Fig. 2 and $2^{\circ}$ in Fig. 2d.

Figures 2c and 2f show typical LDOS measurements recorded in the moiré regions of Figs. 27 and $2 \mathrm{~d}$ respectively. The spectra show resonances which have been attributed to van Hove singularities 20 . These singularities occur due to the interlayer interaction that induces an anticrossing between the two Dirac cones in the electronic dispersion of the two rotated layers $\$ 1621$. Since graphene layers have linear dispersion the energy of this anti-crossing is also linearly dependent on the rotation angle, a property which could be used to tune the van Hove singularities $\frac{1422}{2}$. Figure 2 and 2 show the results 

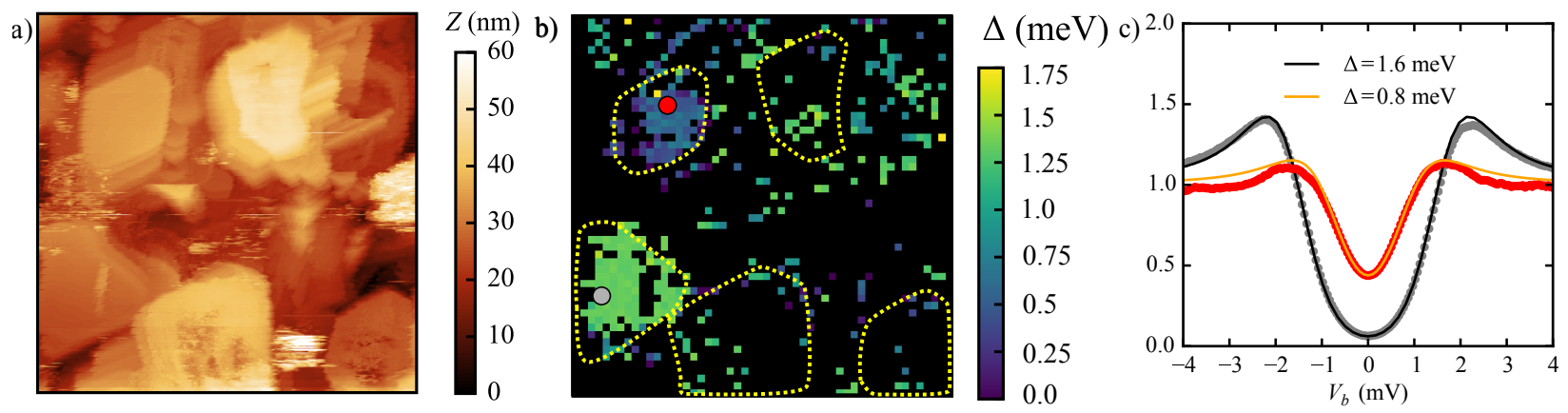

Figure 3. a) A $1 \mu \mathrm{m} \times 1 \mu \mathrm{m}$ STM topography image of the surface of TaC covered with few graphene layers acquired at $2.2 \mathrm{~K}\left(V_{b}=4 \mathrm{mV}\right.$ and $\left.i_{t}=1 \mathrm{nA}\right)$. b) Superconducting gap map in the same region as shown in panel $(a)$. The superconducting gaps were extracted from the $\mathrm{dI} / \mathrm{dV}$ spectroscopy with a $0.1 \mathrm{mV}$ AC bias modulation. Black pixels indicate the places where the superconducting gap could not be estimated. The grains seen in panel a) are highlited with a dotted contour. c) Individual spectra acquired at a position indicated by a dot in panel b) The red dot corresponds to the red spectrum while the gray dot corresponds to the black spectrum. c). Fits to the BCS theory are displayed as solid curves.

of LDOS calculations performed following the tightbinding method (see section IIC). These results reproduce accurately the position of van Hove singularities in the experiments. For the large rotation angle of Fig. 22a the density of states does not depend on the position in the moiré. In contrast, Fig. $2 \mathrm{~d}$ shows more pronounced van Hove singularities in AA regions than in $\mathrm{AB}$ regions for the moiré with a low rotation angle. This stems from the localisation of charge carriers by the moiré potential23. All in all, the rotated graphene layers on $\mathrm{TaC}$ show the same features in the electronic structure as graphene layers on $\mathrm{SiO}^{14 \mid 22}$.

The important conclusion of these STM measurements is that the layers do not grow in the conventional Bernal stacking of graphite but are rotated with respect to each other. Firstly, this suggests that the growth kinetics of graphene on $\mathrm{TaC}$ is fast and resembles that of graphene on the carbon face of $\mathrm{SiC}$ rather that of graphene on the silicon face of $\mathrm{SiC}$. This was suggested by the characteristic shape of the 2D band observed in our previous Raman measurements $\frac{\sqrt{10}}{}$ and finds here a direct confirmation. Secondly and more importantly, in these conditions of rotated growth the electronic dispersion at low energy retains its linear behaviour mimicking that of graphene ${ }^{24}$.

\section{B. Superconductivity of graphene on TaC}

In our previous report we found that the $\mathrm{TaC} /$ graphene film on $\mathrm{SiC}$ becomes superconducting at $T_{c}=10.35 \mathrm{~K}^{10}$. This corresponds to the super- conducting transition temperature of stoichiometric $\mathrm{TaC}^{25}$ and to a superconducting order parameter $\Delta=1.76 k_{B} T_{c}=1.6 \mathrm{meV}$ according to the Bardeen-Cooper-Schrieffer (BCS) theory. Here, we have investigated whether or not the surface of this film is superconducting. The scanning tunnelling spectroscopy gives a direct access to the order parameter at the surface and to its spatial fluctuations by mapping the LDOS at low temperature. Figure $3 \mathrm{~b}$ displays the superconducting gap map. To form this image, a tunnelling spectrum was measured at each pixel and the superconducting gap was measured from each spectra (defective spectra were removed from the analysis). There is a strong correlation between the surface topography (Fig. 3a) and the superconducting gap map. Fitting the experimental data with BCS theory indicates that the superconducting gap varies up to $\Delta=1.6 \mathrm{meV}$ depending on the grain chosen for the measurement. Superconductivity is observed for grains with flat topography while the grains with rougher surface are not. The superconducting gap can vary within a single grain. The graphene surface shows a rich variety of superconducting strength that could result from the following effects or a combination of them. First, all $\mathrm{TaC}$ grains may not be stoichiometric with decreasing $T_{c}$ for off-stoichiometry grains and therefore could present different order parameter amplitudes 25 . However, this explanation is unlikely because the bare presence of multilayers of graphene on top of these grains suggests that the original Ta film has been fully saturated by carbon atoms during the graphene growth. Secondly, since superconductivity is induced by proximity to the 
graphene layers, its strength on the topmost layer depends on the number of layers beneath ${ }^{26}$ and this number may vary significantly from grain to grain. Unfortunately our STM measurements do not allow to discriminate these scenarii. A careful adjustement of the growth condition may allow, if required, to improve the spatial homogeneity of the superconducting properties.

\section{CONCLUSION}

In conclusion a clean and stable superconducting surface can be produced by the graphitization of a tantalum thin film on SiC. Graphene layers do not adopt the Bernal stacking on $\mathrm{TaC}$ but rather grow with a rotational disorder as evidenced by the moiré pattern which preserves the linear dispersion of graphene. Such surface could prove a useful platform for the study of the interaction of superconductivity with magnetic adsorbates in search of topological phases 3 . Its two-dimensional character would promote long-range impurity states, a great asset for visualizing them with scanning probes 27 . In this context, the spatial variation of the superconducting gap between 0 and that of pure TaC could turn into an opportunity for observing at different positions on the same sample the quantum phase transition between a singlet ground state when the superconducting pairing energy is smaller than the Kondo temperature and an unscreened doublet ground state in the opposite situation 28 .
1 W.-T. Pong, C. Durkan, A review and outlook for an anomaly of scanning tunnelling microscopy (stm): superlattices on graphite, Journal of Physics D: Applied Physics 38 (21) (2005) R329.

2 S. De Feyter, F. C. De Schryver, Two-dimensional supramolecular self-assembly probed by scanning tunneling microscopy, Chem. Soc. Rev. 32 (2003) 139150. doi:10.1039/B206566P

3 B. Heinrich, J. Pascual, K. Franke, Single magnetic adsorbates on s-wave superconductors, Prog. Surf. Sci Prog. Surf. Sci. (2018) 1-19. doi:10.1016/j. progsurf.2018.01.001.

${ }^{4}$ O. Fischer, M. Kugler, I. Maggio-Aprile, C. Berthod, C. Renner, Scanning tunneling spectroscopy of hightemperature superconductors, Rev. Mod. Phys. 79 (2007) 353-419. doi:10.1103/RevModPhys.79.353

5 S. Yu, I. Yoshihiro, Highly crystalline 2d superconductors, Nature Rev . Mater 2 (2016) 16094. doi: 10.1038/natrevmats. 2016.94

${ }^{6}$ C. Brun, T. Cren, D. Rodichev, Review of $2 \mathrm{~d}$ superconductivity: the ultimate case of epitaxial monolayers, Supercond. Sci. Technol. 30 (2017) 013003.

7 J. Da, H. Tao, Y. Lixing, L. Qiao, L. Ang, W. Haomin, M. Gang, C. Zhiying, Z. Haoran, Y. Guanghui, Z. Jie, S. Qiujuan, L. Chengtian, X. Hong, X. Xiaoming, J. Mianheng, High-tc superconductivity in ultrathin $\mathrm{bi}_{2} \mathrm{Sr}_{2} \mathrm{Cacu}_{2} \mathrm{O}_{8+x}$ down to half-unit-cell thickness by protection with graphene, Nat. Comm. 5 (2014) 5708. doi:10.1038/ncomms6708

8 Y. Cao, A. Mishchenko, G. L. Yu, E. Khestanova, A. P. Rooney, E. Prestat, A. V. Kretinin, P. Blake, M. B. Shalom, C. Woods, J. Chapman, G. Balakrishnan, I. V. Grigorieva, K. S. Novoselov, B. A. Piot, M. Potemski, K. Watanabe, T. Taniguchi, S. J. Haigh, A. K. Geim, R. V. Gorbachev, Quality heterostructures from two-dimensional crystals unstable in air by their assembly in inert atmosphere, Nano Let- ters 15 (8) (2015) 4914-4921. doi:10.1021/acs. nanolett.5b00648.

${ }^{9}$ C. Tonnoir, A. Kimouche, J. Coraux, L. Magaud, B. Delsol, B. Gilles, C. Chapelier, Induced superconductivity in graphene grown on rhenium, Phys. Rev. Lett. 111 (2013) 246805. doi:10.1103/PhysRevLett. 111.246805

10 T. L. Quang, L. Huder, F. L. Bregolin, A. Artaud, H. Okuno, N. Mollard, S. Pouget, G. Lapertot, C. Chapelier, V. T. Renard, Epitaxial electrical contact to graphene on $\mathrm{SiC}$, Carbon 121 (2017) 48-55. doi:10.1016/j.carbon.2017.05.048

11 T. Aizawa, R. Souda, S. Otani, Y. Ishizawa, C. Oshima, Anomalous bond of monolayer graphite on transition-metal carbide surfaces, Phys. Rev. Lett. 64 (1990) 768-771. doi:10.1103/PhysRevLett.64.768

12 C. Berger, Z. Song, T. Li, X. Li, A. Y. Ogbazghi, R. Feng, Z. Dai, A. N. Marchenkov, E. H. Conrad, P. N. First, W. A. de Heer, Ultrathin epitaxial graphite: $2 \mathrm{~d}$ electron gas properties and a route toward graphene-based nanoelectronics, The Journal of Physical Chemistry B 108 (52) (2004) 19912-19916. doi:10.1021/jp040650f.

13 L. Huder, A. Artaud, T. Le Quang, G. T. de Laissardière, A. G. M. Jansen, G. Lapertot, C. Chapelier, V. T. Renard, Electronic spectrum of twisted graphene layers under heterostrain, Phys. Rev. Lett. 120 (2018) 156405. doi:10.1103/PhysRevLett.120. 156405

14 I. Brihuega, P. Mallet, H. González-Herrero, G. Trambly de Laissardière, M. M. Ugeda, L. Magaud, J. M. Gómez-Rodríguez, F. Ynduráin, J.-Y. Veuillen, Unraveling the intrinsic and robust nature of van hove singularities in twisted bilayer graphene by scanning tunneling microscopy and theoretical analysis, Phys. Rev. Lett. 109 (2012) 196802. doi:10.1103/ PhysRevLett.109.196802 
15 V. Cherkez, G. T. de Laissardière, P. Mallet, J.Y. Veuillen, Van hove singularities in doped twisted graphene bilayers studied by scanning tunneling spectroscopy, Phys. Rev. B 91 (2015) 155428. doi:10. 1103/PhysRevB.91.155428

To G. Trambly de Laissardière, D. Mayou, L. Magaud, Numerical studies of confined states in rotated bilayers of graphene, Phys. Rev. B 86 (2012) 125413. doi:10.1103/PhysRevB.86.125413.

17 A. H. Castro Neto, F. Guinea, N. M. R. Peres, K. S. Novoselov, A. K. Geim, The electronic properties of graphene, Rev. Mod. Phys. 81 (2009) 109-162. doi: 10.1103/RevModPhys.81.109

${ }_{18}$ Z. Y. Rong, P. Kuiper, Electronic effects in scanning tunneling microscopy: Moiré pattern on a graphite surface, Phys. Rev. B 48 (1993) 17427-17431. doi: 10.1103/PhysRevB.48.17427

19 J. M. Campanera, G. Savini, I. Suarez-Martinez, M. I. Heggie, Density functional calculations on the intricacies of moiré patterns on graphite, Phys. Rev. B 75 (2007) 235449. doi:10.1103/PhysRevB.75.235449.

20 L. Van Hove, The occurrence of singularities in the elastic frequency distribution of a crystal, Phys. Rev. 89 (6) (1953) 1189-1193. doi:10.1103/PhysRev.89. 1189

21 J. M. B. Lopes dos Santos, N. M. R. Peres, A. H. Castro Neto, Graphene bilayer with a twist: Electronic structure, Phys. Rev. Lett. 99 (2007) 256802. doi:10.1103/PhysRevLett.99.256802
${ }^{22}$ G. Li, A. Luican, J. M. B. Lopes dos Santos, A. H. Castro Neto, A. Reina, J. Kong, E. Y. Andrei, Observation of Van Hove singularities in twisted graphene layers, Nat. Phys. 6 (2) (2009) 109-113. doi:10. 1038/nphys 1463

${ }^{23}$ G. Trambly de Laissardière, D. Mayou, L. Magaud, Localization of Dirac Electrons in Rotated Graphene Bilayers, Nano Lett. 10 (3) (2010) 804-808. doi:10. $1021 / \mathrm{n} 1902948 \mathrm{~m}$

24 J. Hass, F. Varchon, J. E. Millán-Otoya, M. Sprinkle, N. Sharma, W. A. de Heer, C. Berger, P. N. First, L. Magaud, E. H. Conrad, Why Multilayer Graphene on $4 \mathrm{H}$ - SiC ( 000-1 ) Behaves Like a Single Sheet of Graphene, Phys. Rev. Lett. 100 (12). doi:10.1103/ PhysRevLett.100.125504

25 A. L. Giorgi, E. G. Szklarz, E. K. Stroms, A. L. Bowman, B. T. Matthias, Effect of composition on the superconducting transition temperature carbide and niobium carbide, Phys. Rev. 125 (1962) 837. doi:10.1103/PhysRev.125.837

26 K. D. Usadel, Generalized diffusion equation for superconducting alloys, Phys. Rev. Lett. 25 (1970) 507.

27 G. C. Ménard, et al., Coherent long-range magnetic bound states in a superconductor, Nat. Phys. 11 (2015) 1013-1016. doi:10.1038/nphys3508

28 A. V. Balatsky, I. Vekhter, J.-X. Zhu, Impurityinduced states in conventional and unconventional superconductors, Rev. Mod. Phys. 78 (2006) 373-433. doi:10.1103/RevModPhys.78.373 